\title{
Trend of nutrition research in endocrine disorders, gaps, and future plans: a collection of experiences of an endocrinology research institute
}

\author{
Nazli Namazi ${ }^{1} \cdot$ Rasha Atlasi $^{2} \cdot$ Azadeh Aletaha $^{2} \cdot$ Mojgan Asadi $^{3} \cdot$ Bagher Larijani $^{3}$ \\ Received: 9 October 2020 / Accepted: 8 December 2020 \\ (C) Springer Nature Switzerland AG 2021
}

\begin{abstract}
Background Nutrition plays a pivotal role in the prevention and treatment of endocrine disorders. The aim of this study was to provide a window in order to display the 25-year activities of Endocrinology \& Metabolism Research Institute (EMRI), and the gaps and future plans in the field of nutrition and endocrine disorders.

Methods To collect papers affiliated to the EMRI in field of nutrition from the inception to December 1st 2019, the electronic databases including PubMed/Medline, Web of Science, and Scopus were searched. Publications in English and Persian languages were included. Scientific Landscapes (VOS viewer) software version 1.6.13 was used to provide bibliometric maps.

Results Of 4082 studies identified in the initial search, 319 relevant papers were included. They contributed systematic review and meta-analysis/review $(n=76)$, clinical trials $(n=58)$, cross-sectional $(n=171)$, case-control studies $(n=11)$, and animal studies $(n=3)$. Accordingly, most nutrition studies were dedicated to the level of evidence III (cross-sectional studies: 53.60\%) followed by systematic review studies $(23.82 \%)$ with the level of evidence I. There was also an increasing trend in the nutrition studies through years, with a peak in 2019.

Conclusion An increasing trend in the publications related to nutrition science is observed at EMRI. However, nutrition research and publications can grow further through expanding collaborations with other fields related to endocrine. Given nutritional assessments in national projects and focusing on the identification of preventive nutritional strategies, considering the situations of our society can be helpful to make nutritional findings more practical.
\end{abstract}

Keywords Nutrition $\cdot$ EMRI $\cdot$ Future plan $\cdot$ Endocrine $\cdot$ Publications

\section{Introduction}

Based on the report published by the World Health Organization (WHO), non-communicable diseases (NCDs) represent the leading cause of mortality across

Bagher Larijani

larijanib@tums.ac.ir

1 Diabetes Research Center, Endocrinology and Metabolism Clinical Sciences Institute, Tehran University of Medical Sciences, Tehran, Iran

2 Evidence Based Medicine Research Center, Endocrinology and Metabolism Clinical Sciences Institute, Tehran University of Medical Sciences, Tehran, Iran

3 Endocrinology and Metabolism Research Center, Endocrinology and Metabolism Clinical Sciences Institute, Tehran University of Medical Sciences, Tehran, Iran the world [1]. In the past 20 years, NCD deaths including cardiovascular diseases, diabetes, and obesity have increased by $14.5 \%$ in Iran [2]. Therefore, policy makers need to pay specific attention to identifying preventive strategies in order to reduce economic, social, and psychological burden of such diseases [2,3]. Prevention efforts focus on the four main factors including physical activity, tobacco use, alcohol consumption, and diet [4]. Adherence to healthy dietary patterns and changing from unhealthy eating habits to recommended ones can be helpful in preventing and treating a wide range of diseases including endocrine disorders [5].

Evidence suggests that healthy dietary patterns including Mediterranean diet and Dietary Approaches to Stop Hypertension (DASH) can decrease the risk of various NCDs [6-12]. Healthy diets can also reduce all-cause mortality and mortality attributed to cancer and cardiovascular diseases (CVD) $[6,13,14]$. 
To find preventive strategies to manage endocrine -related diseases, the research centers and groups affiliated to Endocrinology \& Metabolism Research Institute (EMRI) consider nutrition assessments in their research activities. Furthermore, in both professional and public educational programs, nutrition recommendations and their associations with endocrine disorders are considered as well. Although there is no specific nutrition research center at EMRI, accumulating research has been published by researchers affiliated to this institute, particularly in the recent years.

In the present report, we aimed to summarize the 25-year activities of EMRI by focusing on the trend, types of publications, and their remarkable findings in the field of nutrition. Secondary aims were to shed light on nutrition research gaps and suggest a road for the future research, accordingly.

\section{Methods}

To find papers affiliated to the EMRI from the inception to 1 December 2019, PubMed/Medline, Web of Science, and Scopus, the electronic databases were searched. In the present study, we included papers in English or Persian language in the field of nutrition that at least the affiliation of one author was EMRI. Grey literatures including conference papers, theses, letter to editors, and interviews were not included.

All findings were exported to the Endnote library. After removing the duplicates, screening was conducted based on the titles and abstracts to collect all the publications in the field of nutrition. As in this specific issue of journal, there are specific reports on CASPIAN studies, probiotic, Islamic fasting, and osteoporosis, in this study; we did not consider the aforementioned topics to avoid repetition.

Eligible articles were classified based on the type of studies (systematic review, clinical trials, cross-section, case-control, and animal studies). Moreover, the level of evidence for the included studies was determined based on the evidence-based medicine pyramid. In order to clarify the main topics of the nutrition publications, papers were also allocated to either dietary pattern or dietary supplement categories in case of relevancy.

The frequency of publications in each category was expressed as number and/or percent. The trend of publications through years was illustrated as a graph. To provide bibliometric maps, Scientific Landscapes (VOS viewer) software version 1.6.13 was used.

\section{Results}

\section{Literature search}

In total, 8049 papers (duplicate, $n=3951$ ) were identified from PubMed, Scopus, and Web of Science. Initial screening based on titles and abstracts was conducted and 503 nutritional were considered possibly relevant. As publications studies conducted on probiotic, osteoporosis, and Islamic fasting will be explained in other reports, they were excluded from the study to avoid repetition. In the next step, selected papers were checked for affiliations and papers with irrelevant affiliations ( $n=184$ ) were excluded from the study. Finally, we reached 319 papers in the field of nutrition published by researchers affiliated to EMRI.

\section{Main characteristics of the included studies}

The included papers were classified based on the type of studies. They were systematic review and meta-analysis/review $(n=76)$, clinical trials $(n=58)$, cross-sectional $(n=171)$, case-control $(n=11)$, and animal studies $(n=3)$. Most studies were dedicated to secondary research. From the view of evidence-based pyramid, $23.82 \%$ of nutritional studies were placed at the top of the pyramid with the level of evidence I. The most studies had the level of evidence III (53.60\%).

Figure 1 shows the trend of studies conducted in the field of nutrition through years. The first papers with the scope of nutrition were published in 2004. Generally, there was an increasing trend from 2004 to 2019. As shown in Fig. 1, there was a fluctuation in the number of papers between 2004 and 2013. However, after 2013, an increasing trend was observed. Between 2018 and 2019, the number of papers was sharply increased. The most publications were published in the recent 3 years, particularly $2019(n=100)$ (Fig. 1). As shown in Fig. 2, most papers were conducted on obesity and metabolic syndrome. Other frequently used keywords in the title of papers were inflammation, oxidative stress, meta-analysis, health, insulin resistance, overweight, and prevalence. Outstanding authors with high publications in the field of nutrition were Prof. Bagher Larijani, Dr. Ahmad Esmaeilzadeh, and Dr. Leila Azadbakht (Fig. 3). As shown in Fig. 3, the mentioned professors apart from joint papers with each other, have several national and international networks in their publications. In 2019, 32 nutrition papers were dedicated directly to endocrine disorders.

\section{Findings of systematic reviews and meta-analyses}

Based on evidence-based medicine pyramid, the level evidence of systematic review and meta-analysis is "I" and, we only briefly focused on only some results of this type of study.

Systematic review and meta-analysis can be classified into dietary supplements [15-19] and food groups/dietary patterns [20-27]. For instance, cinnamon may be helpful in reducing the serum levels of glucose with no changes in other glycemic parameters and anthropometric indices in patients with diabetes. It can also reduce both systolic and diastolic blood pressures [28]. In addition, its positive effects on obesity measures 
Fig. 1 Trend of publications in the field of nutrition at EMRI

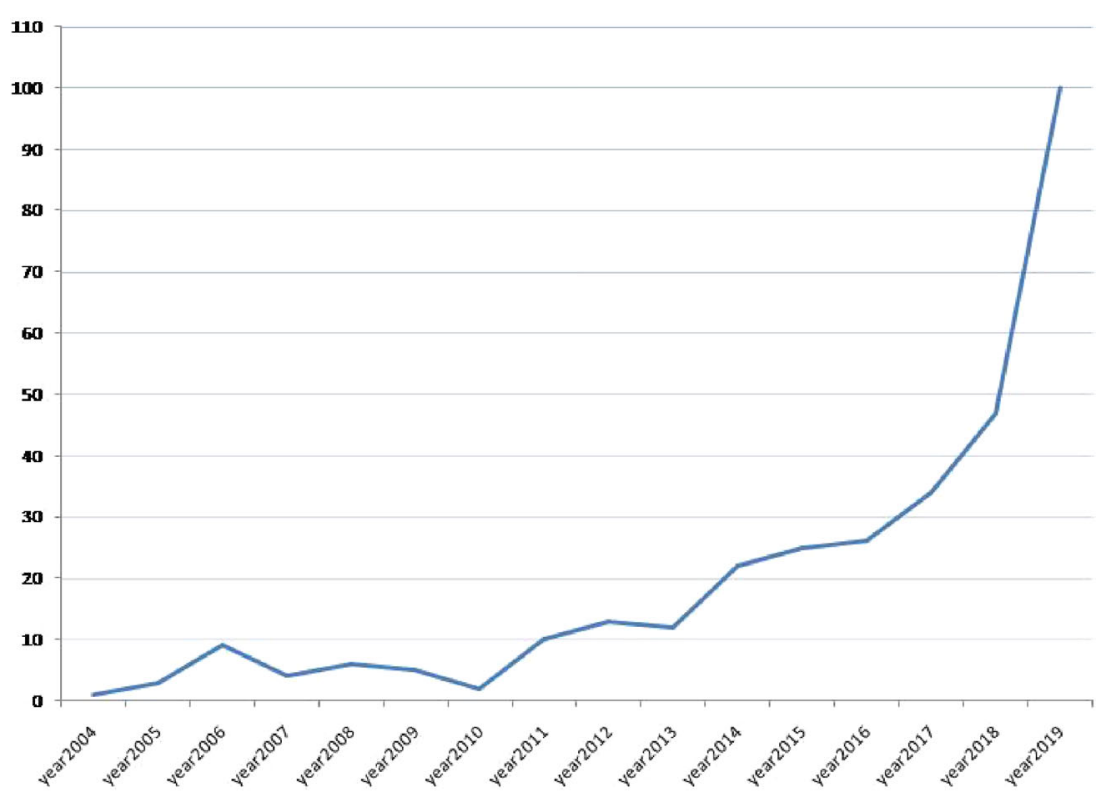

fish oils fetal weight female older-adults fat mass loss stress metabolic syndrome gene-diet interaction insulin sensitivity patterns reduction women glucose fiber serum-cholesterol urban population low density lipoprotein cholesterol osteoprotegerin symptoms inflammation 00 resistance iran humans rate frap obesity pregnancy injuries genotype health impact weight-loss lipoic acid polymorphism type-2 meta-analysis

overweight healthy improves fortification resorption

muscle-cell proliferation

$$
\begin{aligned}
& \text { fat free mass index } \\
& \text { population ffa } \\
& \text { increase progranulin }
\end{aligned}
$$

follow-up neuropeptide

$$
\text { O } 00 \text { men mer }
$$

hip fracture placebo

wc mortality lipid

vitamin-e mood tissue femoral length vitamin-e intervention low-grade inflammation$$
\text { wc men mo }
$$
glycemic index ferritin fruit juice

waist fattyfish vitamin d deficiency waist fatty fish modulation leptin nsulin male herbal medicine sbp gamma gene plasma (c) tea growth physical-activity hypothesis food patterns
vegetables hba1c hd oxidative stress
girls height
vitaminc heart-disease glycemic control prevalence
foods toxicity micronutrient deficiency
middle-east metaanalysis obese adults
hemoglobin mice folic acid

\section{\& Vosviewer}

Fig. 2 Topics of publications in the field of nutrition at EMRI 


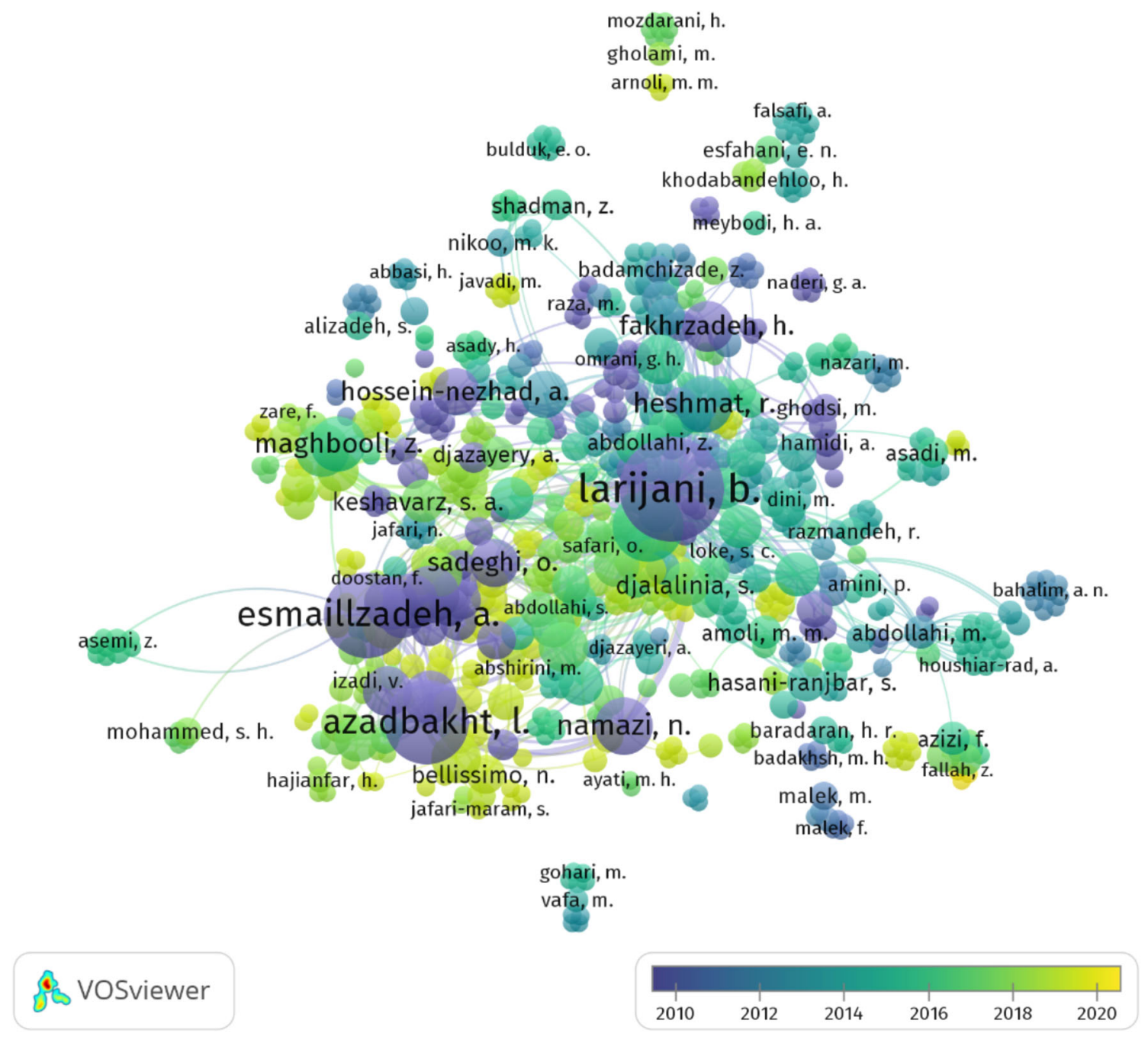

Fig. 3 Outstanding authors in nutrition publications at EMRI

have been revealed [29]. Namazi et al. also concluded that conjugated linoleic acid had positive effects on anthropometric indices and body composition. However, from the clinical points of view, its effects were slight [15]. Regarding supplementation with calcium, it has been shown that it is not effective inreducing serum levels of total cholesterol and triglyceride in overweight and obese individuals. However, it may modulate low-density lipoprotein and high-density lipoprotein cholesterol concentrations [30]. In addition, the consumption of whole-grains did not show any effect anthropometric indices and body composition [20].

\section{Findings of national nutritional research}

Numerous national studies and international studies $[2$, 31-47] with collaboration of European and American countries have been published so far. In this study, we pointed out only some of them as examples.

Rezaei et al., conducted a national study on 18,624 adults and found that the mean salt intake among Iranian population was $9.52 \mathrm{~g}$ /day. In $97.6 \%$ of participants, minimum level of salt consumption was $5 \mathrm{~g}$ /day. Besides, in about $41 \%$ of participants, the level of salt intake was at least twice greater than that recommended by the World Health Organization [44]. Gholami et al. (2016) performed STEPS survey in Iran, and demonstrated that salt intake could increase systolic blood pressure in both Iranian subjects with hypertension and normotensive individuals. However, the magnitude of this increase was greater in hypertensive ones [39].

Obesity is a growing metabolic disorder which has been examined from various aspects. For instance, Djalalinia et al. (2011) found that excess BMI was responsible for 39.5\% of total deaths in subjects (\%55 male, $45 \%$ female) aged 25 to 65 years old at national level. The highest mortality was attributed to ischemic heart diseases $(55.7 \%)$ followed by stroke $(19.3 \%)$ and diabetes mellitus (12.0\%) [35]. Apart from original papers, several systematic reviews and meta-analyses on Iranian studies have been conducted. Based on a systematic review and meta-analysis on 119 studies in Iran, it was revealed that increased age, being married, low level of 
education, residence in urban regions as well as being female were positively associated with obesity [36].

\section{Other activities}

Apart from papers and conference abstracts, some specific activities in the field of nutrition particularly in diabetic research center affiliated to EMRI have been conducted. In the clinical guideline for diabetes published in 2014, a section was dedicated to this field that it was updated in 2018 considering main international guidelines in diabetes as well as national research. The level of evidence has been dedicated to each recommendation in order to help clinicians for making decision. In addition, the road map of diabetes including nutritional section has been provided in 2015 [48, 49] and in 2019 an update based on new evidence has been started.

Furthermore, in several symposiums and conferences held by EMRI including diabetes, osteoporosis, and probiotics, some panels have been dedicated to nutrition. Apart from workshops for physicians and other clinicians, 7 visual educational programs in the field of nutrition and diabetes also have been prepared by cooperation with the visual faculty of Tehran University of Medical sciences, so far. Moreover, numerous booklets and pamphlets in various endocrine disorders, particularly different types of diabetes mellitus, osteoporosis, and elderly disorders have been published and they were updated after a certain period of time to provide recommendations based on new evidence. Several clinics affiliated to EMRI provide nutrition consult and diet therapy for patients. Apart from providing nutrition services for people, they can be suitable ground for doing research in various fields.

\section{Activities in the COVID-19 pandemic}

For adapting to the COVID-19 pandemic, public education in the field of nutrition has been shifted to virtual forms. Animations, motion graphics, E- books, and short films are examples of educational materials that spread through social networks and media by EMRI amid COVID-19. In addition, a guideline on diabetes management in the COVID-19 pandemic is prepared that a specific section has been considered for nutritional recommendations in this challenging time.

\section{Discussion}

Trends of publications in the field of nutrition showed a considerable increase in the recent years at EMRI. Due to the topics of publications, it can be reported that most research centers and groups have considered nutrition assessments in their projects. However, as there is no nutrition research center at EMRI, nutrition roadmap is not completely clear. However, since 2019 a specific group started to develop a roadmap in this field.
Many research projects affiliated to EMRI did not consider nutrition assessments as their main aims; therefore sometimes tools and questionnaires dedicated to this part of projects were not ideal and did not cover the main dimensions of nutrition assessments. Several national research projects have been designed and run by EMRI in collaborations with other institutes including STEPS [50], IMOS [51], Heavy Metal (unpublished protocol), BEHVARZ [52], CASPIAN [53], and Bushehr elderly health program [54] in which nutritional assessments has been considered and some topics on nutritional factors extracted from the aforementioned studies have been published [55-59], but nutrition assessments were only their secondary outcomes. It is suggested that considering specific nutrition aims in such national surveys to draw nutrition status of our society for different age groups and genders, to clarify nutrient deficiencies and other requirements. Based on these findings we can help to policy makers and health providers to develop and implement effective strategies to overcome nutritional problems.

Although the different levels of nutrition research, including international, national, and small studies with various study designs have been conducted at EMRI, more highquality studies are needed to find nutritional requirements and suggest appropriate strategies to prevent and manage endocrine disorders and other NCDs. On the other hand, it seems that paying more attention to basic studies in the field of nutrition can be helpful in developing the effective therapeutic and preventive methods based on nutrition knowledge. It seems that developing studies with several phases from In vitro to clinical trial phase for developing novel dietary supplements and clarification of pathways can be helpful in increasing the numbers of product-based projects.

Based on the publications and activities in the field of nutrition through 25 years, main research gaps in this field related to endocrine disorders are as follows:

(i) No specific strategic plan and action plan in the field of nutrition

(ii) Less attention to basic studies such as animal studies and In vitro studies.

(iii) Undefined priorities in nutrition research

(iv) Less specific attention to nutrition status of study populations and conducting specialized nutrition assessments in national studies conducted by EMRI

(v) Few product-based projects

Given the publications in the field of nutrition, more clinical trials with nutrition topics including different types of diet and dietary supplements were expected. Annual strategic plan and action plan based on the requirements of society, literatures, and the opinion of experts in nutrition sciences can improve the current status. 
Developing multidisciplinary mega projects with practical aims and expanding national and international networks can be considered as a future plan for this research group.Along with increasing the numbers of nutrition projects with highquality methodology, paying attention to hot topics and checking the topics of nutrition research in valid international universities and centers periodically can improve our position in the world. Other proposed future plans in the field of nutrition and endocrine disorders are as follows:

(i) Providing a strategic plan by a professional team in the field of nutrition sciences

(ii) Identifying research gaps in the field of nutrition for each research center particularly for diabetes and obesity research centers to define research priorities

(iii) Focusing on finding preventive strategies for endocrine diseases (national projects considering a collection of nutritional assessments can be helpful)

(iv) Expanding collaborations with experts in the field of basic sciences

(v) Increasing interdisciplinary projects

There were two major limitations in this study that should be addressed. First, the quality of studies was not examined. Second, grey literature such as theses, books, and conference abstracts in the field of nutrition were not considered. The main strengths of this study were as follows: summarizing publications through 25 years in this field, clarifications of research gaps, and putting forward suggestions as future plans.

\section{Conclusion}

An increasing trend in the publications related to nutrition research is observed. However, nutrition activities and publications can grow further through expanding collaborations with other fields related to endocrine. Considering nutritional assessments in national projects and focusing on the identification of preventive nutritional strategies specific to our society can shed light on how to prevent NCDs and decrease the burden of such diseases.

\section{Compliance with ethical standards}

Conflict of interest All authors declared no conflict of interest.

\section{References}

1. World Health Organization. Avaialable at: https://www.who.int/ health-topics/noncommunicable-diseases\#tab=tab_1. 2020.
2. Peykari N, Hashemi H, Dinarvand R, Haji-Aghajani M, Malekzadeh R, Sadrolsadat A, et al. National action plan for noncommunicable diseases prevention and control in Iran; a response to emerging epidemic. J Diabetes Metab Disord. 2017;16(1):3.

3. Khorrami Z, Rezapour M, Etemad K, Yarahmadi S, Khodakarim S, Hezaveh AM, et al. The patterns of non-communicable disease multimorbidity in Iran: a multilevel analysis. Sci Rep. 2020;10(1): $1-11$.

4. Balbus JM, Barouki R, Birnbaum LS, Etzel RA, Gluckman PD, Grandjean P, et al. Early-life prevention of non-communicable diseases. Lancet. 2013;381(9860):3-4.

5. Passi SJ. Prevention of non-communicable diseases by balanced nutrition: population-specific effective public health approaches in developing countries. Curr Diabetes Rev. 2017;13(5):461-76.

6. Onvani S, Haghighatdoost F, Surkan PJ, Larijani B, Azadbakht L. Adherence to the healthy eating index and alternative healthy eating index dietary patterns and mortality from all causes, cardiovascular disease and cancer: a meta-analysis of observational studies. J Hum Nutr Diet. 2017;30(2):216-26.

7. Saneei P, Salehi-Abargouei A, Esmaillzadeh A, Azadbakht L. Influence of dietary approaches to stop hypertension (DASH) diet on blood pressure: a systematic review and meta-analysis on randomized controlled trials. Nutr Metab Cardiovasc Dis. 2014;24(12):1253-61.

8. Arghavani H, Daneshzad E, Namazi N, Larijani B, Askari M, Bellissimo N, et al. Association of adherence to the dietary approach to stop hypertension diet and diet quality indices among women in Tehran: a cross- sectional study. Health Promot Perspect. 2019;9(4):291-8.

9. Ghorabi S, Salari-Moghaddam A, Daneshzad E, Sadeghi O, Azadbakht L, Djafarian K. Association between the DASH diet and metabolic syndrome components in Iranian adults. Diabetes Metab Syndr. 2019;13(3):1699-704.

10. Izadi V, Tehrani H, Haghighatdoost F, Dehghan A, Surkan PJ, Azadbakht L. Adherence to the DASH and Mediterranean diets is associated with decreased risk for gestational diabetes mellitus. Nutr Diet. 2016;32(10):1092-6.

11. Zamani B, Milajerdi A, Tehrani H, Bellissimo N, Brett NR, Azadbakht L. Association of a plant-based dietary pattern in relation to gestational diabetes mellitus. Nutr Diet. 2019;76(5):589-96.

12. Daneshzad E, Emami S, Mofrad MD, Saraf-Bank S, Surkan PJ, Azadbakht L. Association of modified Nordic diet with cardiovascular risk factors among type 2 diabetes patients: a cross-sectional study. J Cardiovasc Thorac Res. 2018;10(3):153-61.

13. Milajerdi A, Namazi N, Larijani B, Azadbakht L. The association of dietary quality indices and cancer mortality: a systematic review and meta-analysis of cohort studies. Nutr Cancer. 2018;70(7): 1091-105.

14. Mohammadifard N, Talaei M, Sadeghi M, Oveisegharan S, Golshahi J, Esmaillzadeh A, et al. Dietary patterns and mortality from cardiovascular disease: Isfahan cohort study. Eur J Clin Nutr. 2017;71(2):252-8.

15. Namazi N, Irandoost P, Larijani B, Azadbakht L. The effects of supplementation with conjugated linoleic acid on anthropometric indices and body composition in overweight and obese subjects: a systematic review and meta-analysis. Crit Rev Food Sci Nutr. 2019;59(17):2720-33.

16. Salari-Moghaddam A, Milajerdi A, Surkan PJ, Larijani B, Esmaillzadeh A. Caffeine, type of coffee, and risk of ovarian cancer: a dose-response meta-analysis of prospective studies. J Clin Endocrinol Metab. 2019;104(11):5349-59.

17. Mousavi SM, Milajerdi A, Sheikhi A, Kord-Varkaneh H, FeinleBisset C, Larijani B, et al. Resveratrol supplementation significantly influences obesity measures: a systematic review and doseresponse meta-analysis of randomized controlled trials. Obes Rev. 2019;20(3):487-98. 
18. Mousavi SM, Milajerdi A, Fatahi S, Rahmani J, Zarezadeh M, Ghaedi E, et al. The effect of L-arginine supplementation on obesity-related indices: a systematic review and Meta-analysis of randomized clinical trials. Anal Chem. 2019;15:1-11.

19. Mofrad MD, Milajerdi A, Koohdani F, Surkan PJ, Azadbakht L. Garlic supplementation reduces circulating C-reactive protein, tumor necrosis factor, and Interleukin-6 in adults: a systematic review and Meta-analysis of randomized controlled trials. J Nutr. 2019;149(4):605-18.

20. Sadeghi O, Sadeghian M, Rahmani S, Maleki V, Larijani B, Esmaillzadeh A. Whole-grain consumption does not affect obesity measures: an updated systematic review and meta-analysis of randomized clinical trials. Turk J Med Sci. 2020;11(2):280-292.

21. Zamani B, Daneshzad E, Azadbakht L. Dietary total antioxidant capacity and risk of gastrointestinal cancers: a systematic review and Meta-analysis of observational studies. Arch Iran Med. 2019;22(6):328-35.

22. Shahdadian F, Saneei P, Milajerdi A, Esmaillzadeh A. Dietary glycemic index, glycemic load, and risk of mortality from all causes and cardiovascular diseases: a systematic review and dose-response meta-analysis of prospective cohort studies. Am J Clin Nutr. 2019;110(4):921-37.

23. Mozaffari H, Daneshzad E, Larijani B, Bellissimo N, Azadbakht L. Dietary intake of fish, n-3 polyunsaturated fatty acids, and risk of inflammatory bowel disease: a systematic review and meta-analysis of observational studies. Nutr Diet. 2019;59:1-17.

24. Malmir H, Larijani B, Esmaillzadeh A. Consumption of milk and dairy products and risk of osteoporosis and hip fracture: a systematic review and Meta-analysis. J Sex Marital Ther. 2020;60(10): $1722-1737$

25. Daneshzad E, Haghighatdoost F, Azadbakht L. Dietary acid load and cardiometabolic risk factors: a systematic review and metaanalysis of observational studies. Public Health Nutr. 2019;22(15):2823-34.

26. Anjom-Shoae J, Sadeghi O, Larijani B, Esmaillzadeh A. Dietary intake and serum levels of trans fatty acids and risk of breast cancer: a systematic review and dose-response meta-analysis of prospective studies. Chem Biodivers. 2020;39(3):755-764.

27. Aminianfar A, Fallah-Moshkani R, Salari-Moghaddam A, Saneei P, Larijani B, Esmaillzadeh A. Egg consumption and risk of upper aero-digestive tract cancers: a systematic review and Meta-analysis of observational studies. Adv Nutr. 2019;10(4):660-72.

28. Namazi N, Khodamoradi K, Khamechi SP, Heshmati J, Ayati MH, Larijani B. The impact of cinnamon on anthropometric indices and glycemic status in patients with type 2 diabetes: a systematic review and meta-analysis of clinical trials. Complement Ther Med. 2019;43:92-101.

29. Mousavi SM, Rahmani J, Kord-Varkaneh H, Sheikhi A, Larijani B, Esmaillzadeh A. Cinnamon supplementation positively affects obesity: a systematic review and dose-response meta-analysis of randomized controlled trials. Clin Nutr. 2020;39(1):123-33.

30. Heshmati J, Sepidarkish M, Namazi N, Shokri F, Yavari M, Fazelian S, et al. Impact of dietary calcium supplement on circulating lipoprotein concentrations and atherogenic indices in overweight and obese individuals: a systematic review. J Diet Suppl. 2019;16(3):357-67.

31. Abarca-Gómez, L., Abdeen, Z. A., Hamid, Z. A., Abu-Rmeileh, N. M., Acosta-Cazares, B., Acuin, C., et al. Worldwide trends in bodymass index, underweight, overweight, and obesity from 1975 to 2016: a pooled analysis of 2416 population-based measurement studies in 128.9 million children, adolescents, and adults. Lancet (London, England). 2017;390(10113):2627-42.

32. Afshin A, Micha R, Khatibzadeh S, Fahimi S, Shi P, Powles J, et al. The impact of dietary habits and metabolic risk factors on cardiovascular and diabetes mortality in countries of the Middle East and
North Africa in 2010: a comparative risk assessment analysis. BMJ Open. 2015;5(5):e006385.

33. Bagheri M, Farzadfar F, Qi L, Yekaninejad MS, Chamari M, Zeleznik OA, et al. Obesity-related Metabolomic profiles and discrimination of metabolically unhealthy obesity. J Proteome Res. 2018;17(4):1452-62.

34. Djalalinia S, Kelishadi R, Qorbani M, Peykari N, Kasaeian A, Nasli-Esfahani E, et al. A systematic review on the prevalence of overweight and obesity, in Iranian children and adolescents. Iran J Pediatr. 2016;26(3):e2599.

35. Djalalinia S, Moghaddam SS, Peykari N, Kasaeian A, Sheidaei A, Mansouri A, et al. Mortality attributable to excess body mass index in Iran: implementation of the comparative risk assessment methodology. Int J Prev Med. 2015;6:107.

36. Djalalinia S, Peykari N, Qorbani M, Larijani B, Farzadfar F. Inequality of obesity and socioeconomic factors in Iran: a systematic review and meta- analyses. Med J Islam Repub Iran. 2015;29: 241.

37. Forouzanfar MH, Alexander L, Anderson HR, Bachman VF, Biryukov S, Brauer M, et al. Global, regional, and national comparative risk assessment of 79 behavioural, environmental and occupational, and metabolic risks or clusters of risks in 188 countries, 1990-2013: a systematic analysis for the Global Burden of Disease Study 2013. Lancet (London, England). 2015;386(10010):2287323.

38. Gholami A, Baradaran HR, Khatibzadeh S, Sheidaei A, Parsaeian M, Farzadfar F. National and subnational cardiovascular diseases mortality attributable to salt consumption in Iran by sex and age from 1990 to 2016. Arch Iran Med. 2018;21(3):122-30.

39. Gholami A, Rezaei S, Jahromi LM, Baradaran HR, Ghanbari A, Djalalinia $\mathrm{S}$, et al. Is salt intake reduction a universal intervention for both normotensive and hypertensive people: a case from Iran STEPS survey 2016. 2020;59:3149-3161

40. Micha R, Khatibzadeh S, Shi P, Andrews KG, Engell RE, Mozaffarian D. Global, regional and national consumption of major food groups in 1990 and 2010: a systematic analysis including 266 country-specific nutrition surveys worldwide. BMJ Open. 2015;5(9):e008705.

41. Micha R, Khatibzadeh S, Shi P, Fahimi S, Lim S, Andrews KG, et al. Global, regional, and national consumption levels of dietary fats and oils in 1990 and 2010: a systematic analysis including 266 country-specific nutrition surveys. BMJ (Clinical research ed). 2014;348:g2272.

42. Ng M, Fleming T, Robinson M, Thomson B, Graetz N, Margono C, et al. Global, regional, and national prevalence of overweight and obesity in children and adults during 1980-2013: a systematic analysis for the Global Burden of Disease Study 2013. Lancet (London, England). 2014;384(9945):766-81.

43. Powles J, Fahimi S, Micha R, Khatibzadeh S, Shi P, Ezzati M, et al. Global, regional and national sodium intakes in 1990 and 2010: a systematic analysis of $24 \mathrm{~h}$ urinary sodium excretion and dietary surveys worldwide. BMJ Open. 2013;3(12):e003733.

44. Rezaei S, Mahmoudi Z, Sheidaei A, Aryan Z, Mahmoudi N, Gohari $\mathrm{K}$, et al. Salt intake among Iranian population: the first national report on salt intake in Iran. Eur J Nutr. 2018;36(12):2380-9.

45. Singh GM, Micha R, Khatibzadeh S, Lim S, Ezzati M, Mozaffarian D. Estimated global, regional, and National Disease Burdens related to sugar-sweetened beverage consumption in 2010. Circulation. 2015;132(8):639-66.

46. Singh GM, Micha R, Khatibzadeh S, Shi P, Lim S, Andrews KG, et al. Global, regional, and National Consumption of sugarsweetened beverages, fruit juices, and Milk: a systematic assessment of beverage intake in 187 countries. PLoS One. 2015;10(8): e0124845. 
47. Stevens GA, Singh GM, Lu Y, Danaei G, Lin JK, Finucane MM, et al. National, regional, and global trends in adult overweight and obesity prevalences. Popul Health Metrics. 2012;10(1):22.

48. Shafiee G, Bandarian F, Ghodsi M, Nasli-Esfahani E, Larijani B. Iran Diabetes Research Roadmap (IDRR) study; trends of publications in management of diabetes in Iran: a review article. Iran J Public Health. 2017;46(Supple 1):3-9.

49. Ebrahimi ZS, Peimani M, Azadbakht L, Nasli-Esfahani E, Farideh R, Larijani B. Iran diabetes research roadmap (IDRR) study; nutrition in diabetes: a review article. Iran J Public Health. 2017;46(Supple 1):17-25.

50. Djalalinia S, Modirian M, Sheidaei A, Yoosefi M, Zokaiee H, Damirchilu B, et al. Protocol design for large-scale cross-sectional studies of surveillance of risk factors of non-communicable diseases in Iran: STEPs 2016. Arch Iran Med. 2017;20(9):608-16.

51. Meybodi HA, Heshmat R, Maasoumi Z, Soltani A, HosseinNezhad A, Keshtkar A, et al. Iranian osteoporosis research network: background, mission and its role in osteoporosis management. Iran J Public Health. 2008;37(supp):1-6.

52. Farzadfar F, Murray CJ, Gakidou E, Bossert T, Namdaritabar H, Alikhani S, et al. Effectiveness of diabetes and hypertension management by rural primary health-care workers (Behvarz workers) in Iran: a nationally representative observational study. Lancet. 2012;379(9810):47-54.

53. Azemati B, Heshmat R, Qorbani M, Ahadi Z, Azemati A, Shafiee $\mathrm{G}$, et al. Association of meal skipping with subjective health complaints in children and adolescents: the CASPIAN-V study. Eat Weight Disord. 2020;25(1):241-6.
54. Ostovar A, Nabipour I, Larijani B, Heshmat R, Darabi H, Vahdat K, et al. Bushehr elderly health (BEH) Programme, phase I (cardiovascular system). BMJ Open. 2015;5(12):e009597.

55. Azemati B, Kelishadi R, Ahadi Z, Shafiee G, Taheri M, Ziaodini H, et al. Association between junk food consumption and cardiometabolic risk factors in a national sample of Iranian children and adolescents population: the CASPIAN-V study. Eat Weight Disord. 2020;25(2):329-35.

56. Bagherzadeh M, Sajjadi-Jazi S, Sharifi F, Ebrahimpur M, Amininezhad F, Ostovar A, et al. Effects of metabolic syndrome on bone health in older adults: the Bushehr elderly health (BEH) program.Osteoporos Int. 2020;31:1975-84.

57. Hossein-Nezhad A, Khoshniat N, Maghbooli M, Karimi Z, Mirzaei F, Hosseini A, et al. Relationship between serum vitamin D concentration and metabolic syndrome among Iranian adults population. DARU. 2015;1:1-5.

58. Pourrostami K, Heshmat R, Hemati Z, Heidari-Beni M, Qorbani M, Motlagh ME, et al. Association of fruit and vegetable intake with meal skipping in children and adolescents: the CASPIAN-V study. Eat Weight Disord. 2020;25(4):903-10.

59. Azizi-Soleiman F, Khoshhali M, Heidari-Beni M, Qorbani M, Pourmirzaei MA, Kelishadi R. Higher dietary phytochemical index is associated with anthropometric indices in children and adolescents: the weight disorders survey of the CASPIAN-IV study. Int J Vitam Nutr Res. 2020;10: 1-8.

Publisher's note Springer Nature remains neutral with regard to jurisdictional claims in published maps and institutional affiliations. 